\title{
The Impact of Thyroid Autoantibodies Positivity on In Vitro Fertilization Outcome: A Comprehensive Review
}

\author{
Sokratis Grigoriadis ${ }^{1}$, Evangelos Maziotis ${ }^{1}$, Mara Simopoulou ${ }^{1,2^{*}}$, Konstantinos Sfakianoudis ${ }^{3}$, Polina \\ Giannelou ${ }^{1,3}$, Anna Rapani ${ }^{1}$, Petroula Tsioulou ${ }^{1}$, Agni Pantou $^{2}$, Despina Tzanakaki ${ }^{2}$, Konstantinos \\ Pantos $^{3}$ and Michael Koutsilieris ${ }^{1}$
}

\begin{abstract}
${ }^{1}$ Department of Physiology, Medical School, National and Kapodistrian University of Athens, Greece
${ }^{2}$ Assisted Conception Unit, $2^{\text {nd }}$ Department of Obstetrics and Gynecology, Aretaieion Hospital, Medical School, National and Kapodistrian University of Athens, Greece

${ }^{3}$ Centre for Human Reproduction, Genesis Athens Clinic, Greece
\end{abstract}

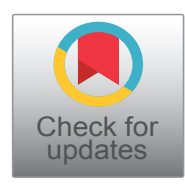

*Corresponding author: Mara Simopoulou, Assistant Professor of Physiology, Clinical Embryologist/Geneticist, Department of Physiology; Department of Obstetrics and Gynaecology, Medical School, National and Kapodistrian University of Athens 75, Mikras Asias 11527, Athens, Greece, Tel: +302107462592 , Fax: +302107462571

\section{Abstract}

Background: In Vitro Fertilization (IVF) has been employed for more than four decades. A crucial concern is the etiology of infertility due to the fact that it may range widely, from reproductive system anatomy and endometrium receptivity to multisystem disorders and several immunologic factors. Among the immune pathologies that could compromise fertility are the autoantibodies affecting thyroid function. Infertile women are in a great prevalence of thyroid autoimmune disorders. However, there are still unsatisfactory data regarding the direct impact of thyroid autoantibodies (TAA) on IVF outcome. The aim of this study is to provide a comprehensive review of the impact of thyroid autoantibodies on the possibilities of a successful IVF cycle outcome.

Methods: PubMed/Medline, Embase and Cochrane Central databases were thoroughly searched. Only fourteen studies were assessed as eligible for the present comprehensive review, and their design and outcome measures -namely clinical pregnancy, live birth rate, and miscarriage rate- are presented herein.

Results: The great majority of the published studies demonstrated that TAA presence in euthyroid women does not appear to be associated with statistically significant different outcomes following IVF treatment. A trend towards a higher miscarriage rate in TAA positive women was reported in several studies. However, this observation was not confirmed by other studies. In addition, studies presented with a high heterogeneity hence robust conclusions could not be safely sourced.
\end{abstract}

Conclusion: In conclusion, well-designed prospective cohort studies are imperative in order to define the direct impact of TAA on IVF cycle success. The contribution of future studies is of significance especially in light of the fact that autoantibodies and their role in infertility treatment is still be undervalued, and underrepresented, as evident by the lack of data published on this complex field.

\section{Keywords}

Thyroid, Thyroid Auto-Antibodies, Autoimmune Disorders, Assisted Reproduction, In Vitro Fertilization

\begin{abstract}
Abbreviations
IVF: In Vitro Fertilization; ART: Assisted Reproductive Techniques; HPT: Hypothalamic-Pituitary-Thyroid; HPO: Hypothalamic-Pituitary-Ovarian; TAA: Thyroid Autoantibody; TSH: Thyroid Stimulating Hormone; Tg-Abs: Thyroid Globulin Auto-Antibodies; anti-TPO: Anti-Thyroperoxidase antibodies; ICSI: Intra Cytoplasmic Sperm Injection
\end{abstract}

\section{Introduction}

In Vitro Fertilization (IVF) is widely considered as one of the most recognizable scientific achievements of the $20^{\text {th }}$ century [1] and its employment has increased in the last 40 years to the point that according to estimations at least seven million babies have been born from couples undergoing Assisted Reproductive

\footnotetext{
Citation: Grigoriadis S, Maziotis E, Simopoulou M, Sfakianoudis K, Giannelou P, et al. (2019) The Impact of Thyroid Autoantibodies Positivity on In Vitro Fertilization Outcome: A Comprehensive Review. Int Arch Clin Physiol 1:002.

Accepted: March 26, 2019: Published: March 28, 2019

Copyright: (c) 2019 Grigoriadis S, et al. This is an open-access article distributed under the terms of the Creative Commons Attribution License, which permits unrestricted use, distribution, and reproduction in any medium, provided the original author and source are credited.
} 
Techniques (ART). A constant concern as viewed by both clinicians and patients is the correct and timely diagnosis of the infertility factor involved. This could lead to correct decision making regarding the optimal treatment approach. Infertility etiology ranges widely, from uterine anatomy and endometrium receptivity to connective tissue disorders and several immunologic factors, hindering the possibilities of a successful IVF cycle. One of the most common autoimmune disorders affecting women of reproductive age is thyroid autoimmunity, detected by thyroid autoantibodies.

The Hypothalamic-Pituitary-Thyroid (HPT) axis and the Hypothalamic-Pituitary-Ovarian (HPO) axis interact with each other. Numerous thyroid hormone receptors that exist in the ovaries regulate the physiological interaction between the two aforementioned axes. It is suggested that estrogen directly affects the HPT axis functionality on the hypothalamus-pituitary level [2]. Menstrual disturbances and anovulatory cycles, affect women that are diagnosed either with hypothyroidism or hyperthyroidism.

According to prevalence studies women diagnosed with thyroid autoimmunity, namely Grave's autoimmunity and Hashimoto disease, were also diagnosed as infertile at the rates of $47 \%$ and $52 \%$, respectively [3]. Thyroid autoantibody (TAA) levels were statistically significantly higher in infertile women (19\%) compared to fertile women (13\%) according to a recent cross-over study. Furthermore, serum Thyroid Stimulating Hormone (TSH) and Thyroid Globulin Auto-Antibodies (TgAbs) levels were statistically significantly higher in women with thyroid auto-immunity presented compared to a control group [4,5]. It is suggested by other studies that anti-Thyroperoxidase antibodies (anti-TPO) are correlated with infertility in age-matched women, regardless of possible euthyroid status [6]. Furthermore, a possible correlation between thyroid autoimmunity and infertility is suggested. However, there are no indications for a causative relationship between thyroid auto-immunity and infertility leading the practitioners to several dilemmas regarding the appropriate management of Thyroid Auto-Antibodies (TAA) positive infertile women that are undergoing ART [7].

The aim of this study is to investigate a possible correlation between IVF outcome and thyroid autoimmunity and present the current published literature in order to provide information regarding the IVF cycle outcome of women diagnosed positive for thyroid autoimmunity.

\section{Methods}

A thorough search of the literature was performed on December $1^{\text {st }}, 2018$, from 2006 to date, on PubMed/ Medline database, Embase database, and Cochrane Central database. It is well established in the literature that a continuous improvement on IVF success rates is documented from 1978 till 2006, as indicated from the
Table 1: Search Strategy.

\begin{tabular}{|l|l|}
\hline 1 & In Vitro Fertilization \\
\hline 2 & Intracellular Sperm Injection \\
\hline 3 & Intrauterine insemination \\
\hline 4 & IVF \\
\hline 5 & ICSI \\
\hline 6 & IUI \\
\hline 7 & Assisted Reproduction \\
\hline 8 & \# 1 OR \# 2 OR \# 3 $\ldots$. \\
\hline 9 & Immune system \\
\hline 10 & Immune disorders \\
\hline 11 & Antibodies \\
\hline 12 & Autoantibodies \\
\hline 13 & Autoimmune \\
\hline 14 & Anti-nuclear antibodies \\
\hline 15 & Antisperm antibodies \\
\hline 16 & \# 9 OR \# 10.... \\
\hline 17 & \# 8 AND \# 16 \\
\hline
\end{tabular}

higher live birth rates throughout the aforementioned years [8]. This continuous improvement seems to reach a plateau in 2006, and since then, live birth rates are at the same level with minor adjustments reported each year. In light of that, only studies that were performed following 2006 were considered suitable to be included in this review. The keywords employed and combined for the search strategy are presented in (Table 1 ).

In several studies, the possible impact of different types of auto-antibodies on IVF outcome was investigated. Thus, the key-words employed in this review generically represent the field of ART, under the context of auto-immune disorders, in an effort to include all relevant articles. Further to that, the search terms were designed to include full texts of each article instead of limiting the search to titles or abstracts, or keywords. The search was limited to full-length manuscripts published in English, in peer-reviewed journals up to December 2018 , in which the possible effect of TAA on IVF/ICSI outcome was investigated. These criteria resulted in the identification of 14 peer-reviewed articles yielded from 598 studies identified in the primary search. Two authors independently screened the literature and selected the appropriate studies with respect to the inclusion criteria.

\section{Results}

The literature search revealed fourteen studies investigating the possible impact of thyroid autoimmunity on IVF outcome. All these studies met the inclusion criteria and were considered suitable for inclusion in this review [9-22]. Information regarding the studies' characteristics namely the year of publication, the type of methodology employed in each study and the type of TAA investigated are reported and discussed throughout 


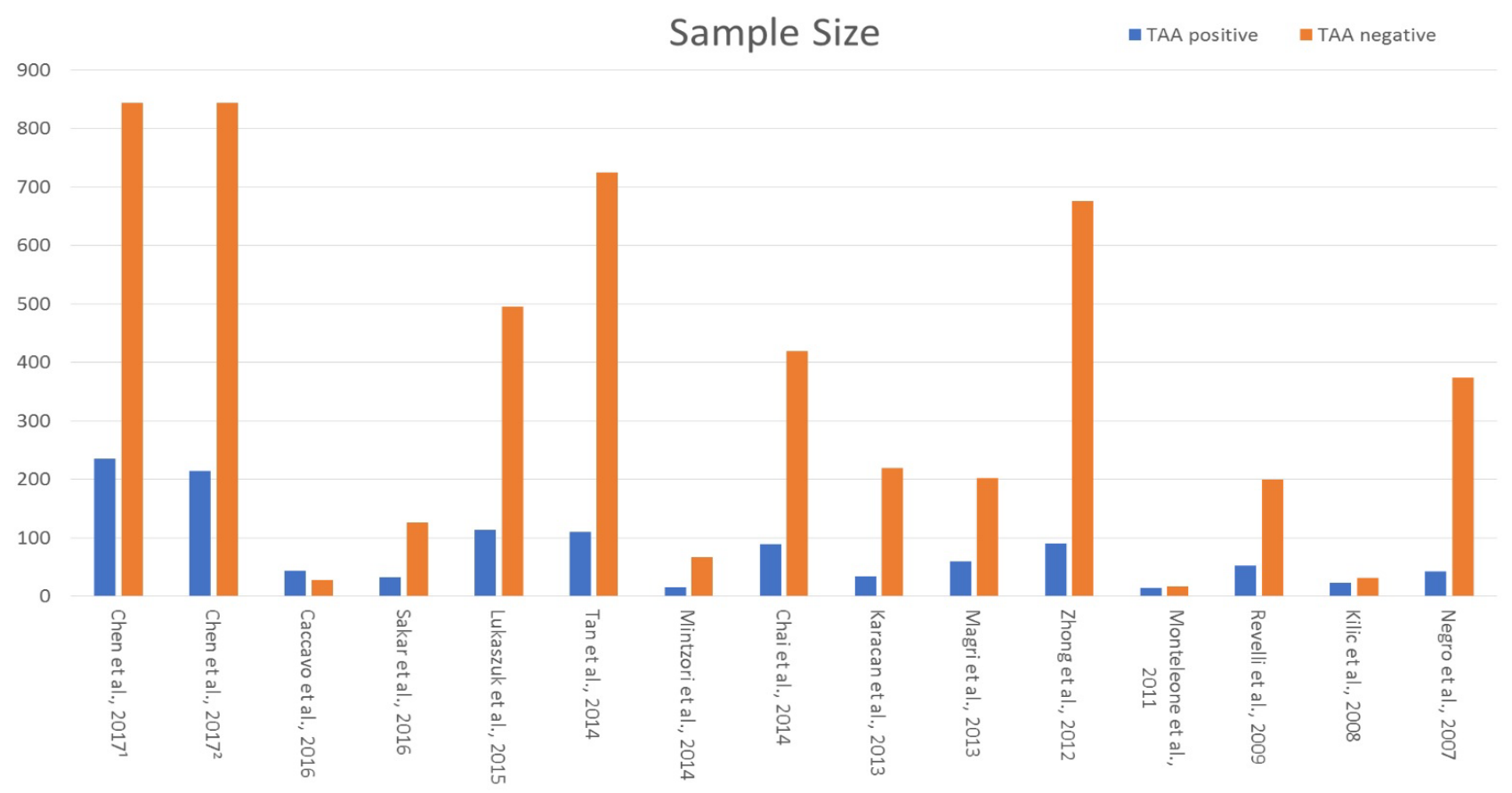

Figure 1: Sample size of studies populations [8,11-24].

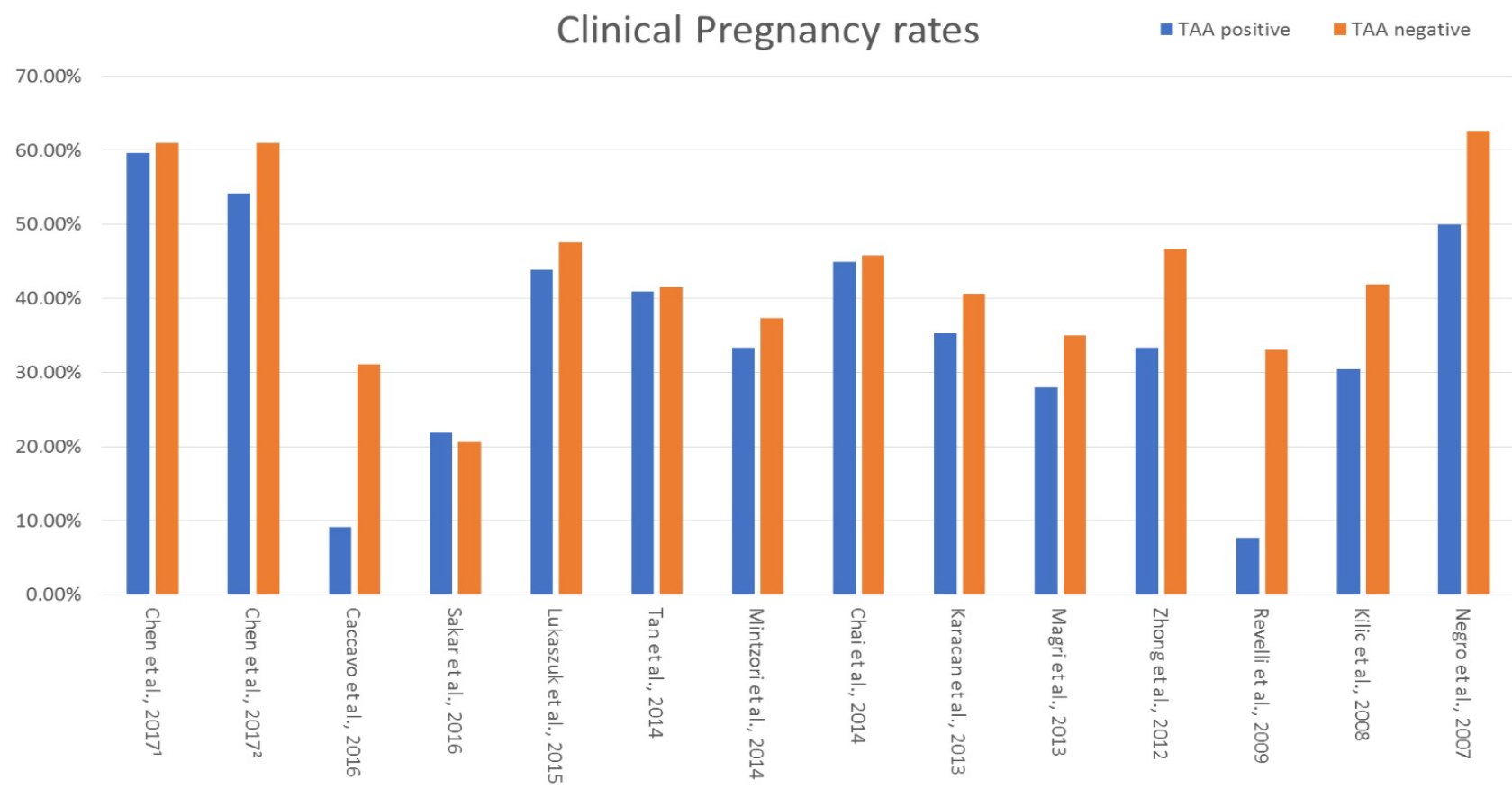

Figure 2: Clinical Pregnancy Rates presented in each study [8,11-18,20-24].

the manuscript. Data regarding the size of the studied population and the IVF outcomes presented in each study, namely the clinical pregnancy rate, the live birth rate, and the miscarriage rate are presented throughout the text and also presented in (Figures 1, Figure 2, Figure 3 and Figure 4). The eight out of the fourteen studies included in this review were of retrospective nature and the other six performed a prospective cohort analyses.

Prospective studies investigating the impact of thyroid auto-antibodies on IVF/ICSI outcome
In the first study, Chen, et al. [11] prospectively investigated the impact of TAA on IVF/ICSI outcome. Two hundred fifteen women (215) with positive Tg-Ab and two hundred fourteen (214) with positive TPO-Ab antibodies were compared to 844 women negative for auto-antibodies. Tg-Ab and TPO-Ab positive groups were presented with no statistically significant differences regarding clinical pregnancy rate, miscarriage rate, live birth rate/ongoing pregnancy rate compared to TAA negative women. TPO-Ab positive women presented with lower implantation rate in comparison to both $\mathrm{Tg}-\mathrm{Ab}$ positive group and TAA negative group. Data provided from this 


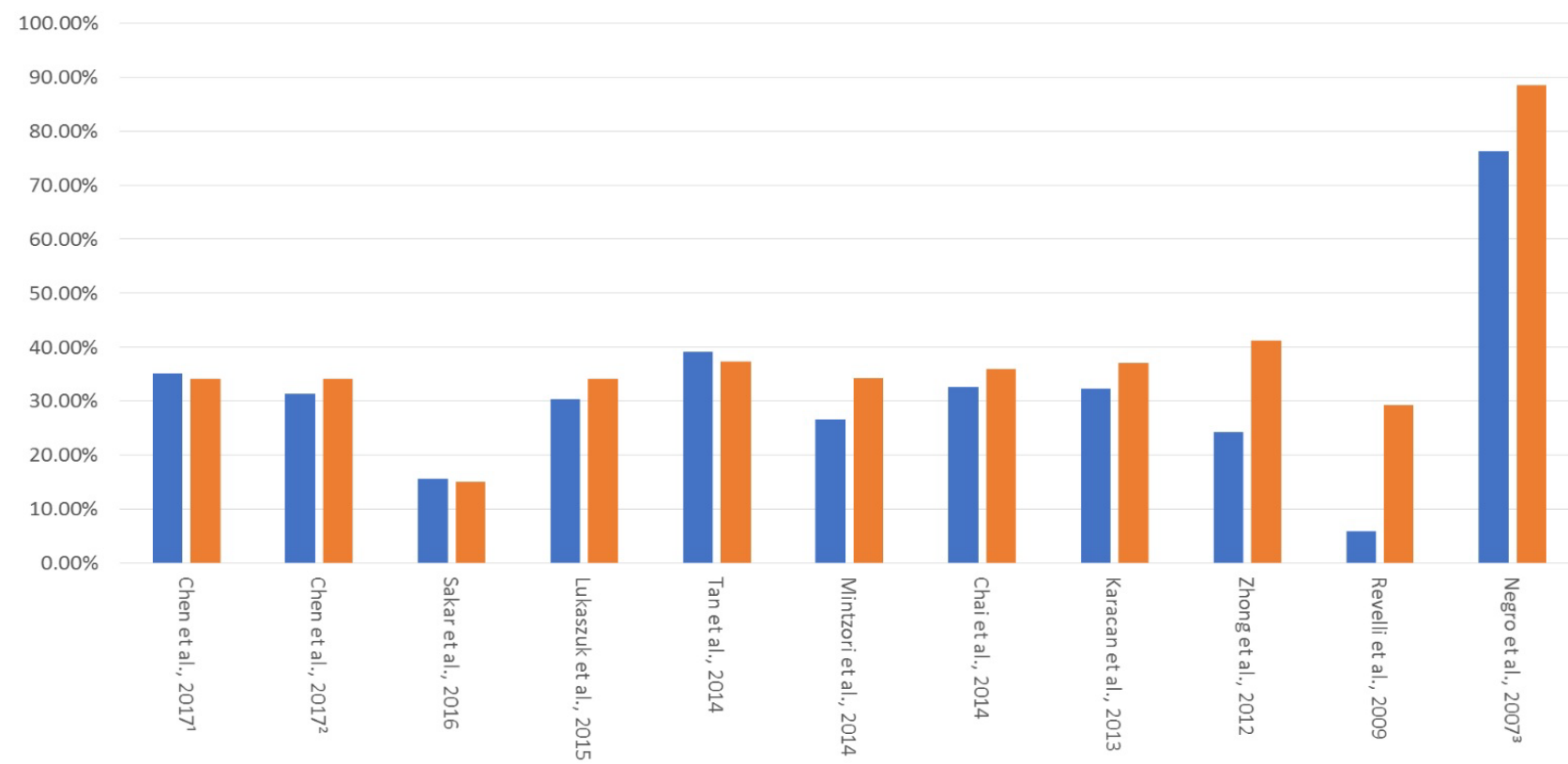

Figure 3: Live Birth Rates presented in each study [8,12-14,16,18,20-24].

\section{Miscarriage rates}

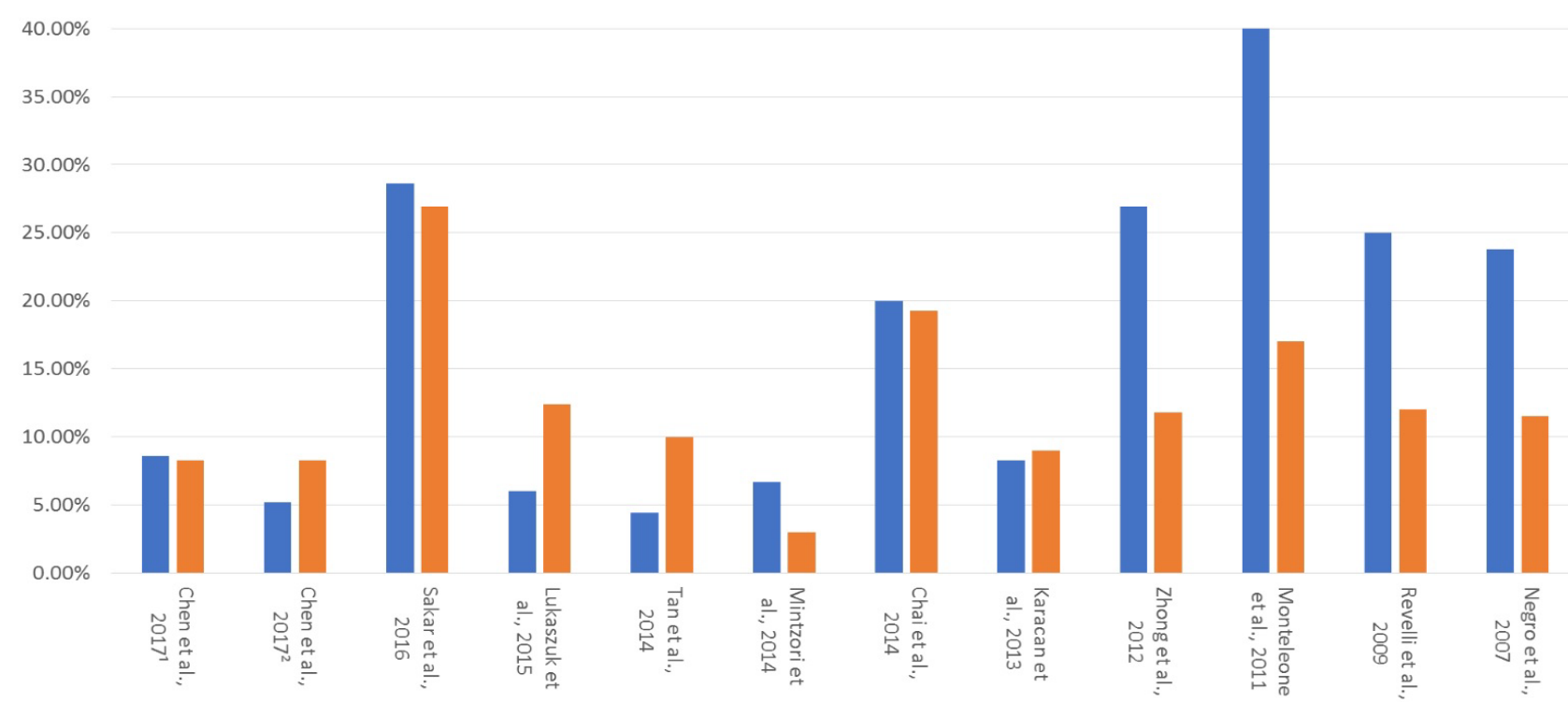

Figure 4: Miscarriage Rates presented in each study [8,12-14,16,18-24].

study are in the line that TAA positivity could not impair significantly IVF outcome. Caccavo, et al. [9] investigated the impact of Anti-laminin-1 antibodies in serum and follicular fluid of women affected by Hashimoto's thyroiditis on IVF outcome. The study group consisted of 44 infertile women with Hashimoto's thyroiditis submitted to controlled ovarian stimulation and IVF due to male factor or tubal factor infertility only. Hashimoto's thyroiditis diagnosis was performed by standard clinical and laboratory criteria, including measures of TPO-Ab, $T g-A b$ in patients' sera. The control group consisted of 28 age-matched infertile women without Hashimoto's thyroiditis submitted to controlled ovarian stimulation and IVF due to male factor or tubal factor infertility. Women with Hashimoto's thyroiditis presented with statistically significant elevated levels of TPO-Ab, Tg-Ab and Antilaminin-1 antibodies in comparison to controls. Further to that, implantation and clinical pregnancy rates were statistically significantly lower in women with Hashimoto's thyroiditis in comparison to women without $(7.9 \%$ vs. $23 \%, 9.1 \%$ vs. $31.1 \%$, respectively). The fertilization rate did not differ significantly between the two groups (67.4\% vs. $73.4 \%)$. Additionally, Anti-laminin-1 antibodies presented to be negatively cor- 
related with the number of MII oocytes retrieved. Authors concluded that Anti-laminin-1 antibodies in correlation with TAA could impair IVF outcome in women with Hashimoto's thyroiditis. Respecting the data provided from this study, conclusions regarding the direct impact of the TAA on IVF outcome in women with Hashimoto's disease could not be drawn due to the presence of additional comorbidities acting as confounding factors. In a prospective single-blind case-control study published by Sakar, et al. [20] the impact of TAA on ICSI-ET cycles was evaluated. All study participants were euthyroid women and selected randomly by the simple random method. Participants were divided into two groups, namely the TAA (+) group (31 women) and the TAA (-) group (121 women), with regards to the presence or not of TAA in women sera. No statistically significant differences could be established between TAA (+) and TAA (-) groups regarding the patients' age, the etiology of infertility, the hormonal profiling, the thyroid functionality, the response to ovarian stimulation and the number of oocytes collected. In addition to the abovementioned parameters, the fertilization, the implantation, and the clinical pregnancy rates did not differ significantly between the two groups (50.8\% vs. $57.5 \%, 30 \%$ vs. $31.6 \%, 21.90 \%$ vs. $20.6 \%$ ). The results were similar in terms of the miscarriage rate and the ongoing pregnancy rate between the TAA $(+)$ group and the TAA (-) group and no statistically significant difference was observed ( $28.6 \%$ vs. $26.9 \%, 15.6 \%$ vs. $15.1 \%$ ). In contrast to the study published by Caccavo, et al. [9] in which the study group consisted of women with Hashimoto's thyroiditis, in this study, authors decided to include randomly only euthyroid women in an effort to dismiss all possible confounders. Regarding these results, TAA per se does not impair the IVF/ICSI outcome. However, and as the authors conclude, this study was characterized by a limited sample size posing as a serious limitation. In a separate prospective study published by Karacan, et al. [12], the effect of TAA on ICSI outcome was investigated in euthyroid women negative for anticardiolipin antibodies and lupus anticoagulant. As in the study of Sakar, et al. [20], the participants of this study were divided into two groups namely TAA (+) group and the TAA (-) group. No statistically significant differences were observed between the two groups regarding the patients' age, the etiology of infertility, the thyroid functionality, the response to ovarian stimulation and the number of oocytes retrieved. Further to that, no statistically significant difference could be established between the TAA (+) and the TAA (-) group concerning the fertilization rate $(78.7 \%$ vs. $79.7 \%)$, the implantation rate $(18.4 \%$ vs. $19.1 \%)$, the clinical pregnancy rate (35.3\% vs. $40.6 \%)$, the ongoing pregnancy rate $(32.4 \%$ vs. $37 \%)$ and the miscarriage rate ( $8.3 \%$ vs. $9 \%)$. Results presented in this study were similar to the results were provided by Sakar, et al. [20], indicating that the presence of TAA does not significantly impair IVF outcome.
Nevertheless, the power of the evidence provided is limited by the small size of the participants in the TAA $(+)$ group. Contradictory to the results already discussed $[12,20]$ are the results sourced by a small prospective observational study published by Monteleone, et al. [17]. In this study, 14 euthyroid women with thyroid autoimmunity (TAA (+)) presenting with lower fertilization and implantation rates compared to 17 euthyroid TAA (-) women (63\% vs. $72 \%, 29 \%$ vs. $43 \%$ ), but only the difference in the fertilization rate was statically significant. In addition, TAA (+) women presented with a lower number of good quality embryos ( $25 \%$ vs. $48 \%$ ) and higher early miscarriage rates ( $40 \%$ vs. $17 \%$ ) in comparison to the TAA (-) control group. However, these differences failed to establish statistical significance. An observation of high significance documented in this study for the first time in literature is the presence of TAA in the follicular fluid of TAA (+) women. In addition to that, the finding of TAA levels in the follicular fluid was presented to be of strong linear correlation to the levels of TAA in the sera of TAA (+) women. Authors concluded that TAA may affect negatively the IVF outcome by directly compromising the ovarian function. Based on the study characteristics, such a conclusion cannot be established with safety, mainly due to the very small number of the individuals included in the study population. Further to that, TAA $(+)$ women presented with elevated serum TSH levels $(2.5 \pm 1.2 \mu \mathrm{U} / \mathrm{ml})$, a parameter which indicates that these individuals were borderline subclinical hypothyroidism. It is well documented that subclinical hypothyroidism could adversely affect IVF treatment per se, in a dose-related fashion. Thus, it is recommended that women with subclinical hypothyroidism should be treated with levothyroxine (LT4) prior to IVF/ ICSI treatment in order to reduce TSH level in concentrations $<2.5 \mu \mathrm{U} / \mathrm{ml}$ [7]. Additional data, similar to those that have been presented by the studies of Sakar, et al. [20] and Karacan, et al. [12], are provided by the study of Kilic, et al. [13]. In this cross-sectional and prospective study, 31 euthyroid TAA (-) women were compared to 23 euthyroid TAA $(+)$ women and 15 TAA $(+)$ women who were euthyroid employing medical treatment. The three groups did not differ significantly in regard to the patients' age, the menstrual cycle hormonal profile and the etiology of infertility. The number of oocytes retrieved, the number of miscarriages, the fertilization rate and the quality of embryos transferred were similar among the three groups. Regarding implantation rate (in this study this is referred to as biochemical pregnancy rate) no statistically significant difference could be established. Clinical pregnancy rates were significantly differentiated among the three groups (41.9\% vs. $30.4 \%$ vs. $13.4 \%)$. The cause of this significant difference was the group of the TAA (+) women who were euthyroid employing medical treatment, as a subgroup analysis revealed, indicating that the clinical pregnancy rate did not differ significantly among the euthyroid TAA (-) 
women and the euthyroid without medical treatment TAA (+) women. In addition, no linear correlation could be established between the levels of the TAA and the fertilization rate in the euthyroid TAA (-) group and in the euthyroid without medical treatment TAA (+) group. A statistically significant negative correlation could be established only between the levels of the TPO-Ab and the fertilization rate in the group of TAA $(+)$ women who were euthyroid with medical treatment. In the same study, the predictive value of the TAA positivity on clinical pregnancy was investigated. Only the presence of TPO-Ab was evaluated as significant. The cut-off point for the prediction was set out at $69.78 \mathrm{IU} / \mathrm{ml}$. Although the Negative Predictive Value (NPV) was high (85.75\%), the Positive Predictive Value (PPV) presented to be very low (44.05\%). Authors conclude that TPO-Ab positivity could impair IVF outcome by reducing the clinical pregnancy rate. This correlation could be established only in the group of TAA $(+)$ women who were euthyroid with medical treatment. For this reason, conclusions regarding the direct impact of the TAA on IVF outcome in women who are euthyroid without medical treatment could not be safely drawn with respect to the data provided from this study. Further to that, the limited sample size of the participants, especially regarding the group of the TAA (+) women who were euthyroid with medical treatment, poses a serious limitation.

\section{Retrospective studies investigating the impact of thyroid auto-antibodies on IVF/ICSI outcome}

Concerning the retrospective studies evaluated in this review, the great majority of them are in favor of the view that the TAA could not impair the IVF outcome per se. In a study in 2015 published by Lukaszuk, et al. [14], 114 euthyroid TPO-Ab (+) women were compared to 495 euthyroid TPO-Ab (-) women in respect to the IVF outcome. No statistically significant difference could be established regarding fertilization rate $(65.1 \%$ vs. $60.5 \%)$, implantation rate $(25.11 \%$ vs. $25.28 \%)$, clinical pregnancy rate $(43.8 \%$ vs. $47.5 \%)$, live birth rate $(30.4 \%$ vs. $34.1 \%$ ) and miscarriage rate (6\% vs. $12.4 \%$ ). Additionally, the patients' age, the hormonal profile (FSH, $\mathrm{LH}, \mathrm{AMH}$ and E2 levels), the etiology of infertility, the response to ovarian stimulation, the number of oocytes retrieved, and the number of transferred embryos did not differ significantly between the two groups. In the same line to the study of Lukaszuk, et al. [14], was the study published a year before by Tan, et al. [21]. In this study, 835 healthy euthyroid women underdoing ICSI due to male factor infertility were included. From the 835 women included in the study, 110 women were TAA (+) (defined as TPOAb or Tg-Ab > $100 \mathrm{U} / \mathrm{I}$ ) and the remaining 725 were TAA (-). No statistically significant difference could be established with respect to the patients' age, the clinical pregnancy rate (OR: 1.00 (95\% $\mathrm{Cl}: 0.66-1.52))$ and the live birth rate (OR: $1.10(95 \% \mathrm{Cl}$ : 0.72-1.66)) between the two groups. Authors concluded that the TAA per se does not negatively affect the ICSI outcome in a proved cohort of euthyroid healthy women. This study is of particularly importance as authors dismissed any parameters that could knowingly hinder the positive IVF outcome, and hence act as confounders. They employed a study methodology in which all women were otherwise healthy-only couples with male factor infertility were included in the study- and were matched according to their age. Results presented in a study published by Negro, et al. [18], have shown that TPO-Ab (+) euthyroid women achieve the same clinical pregnancy and live birth rates to those who were TPO-Ab (-). In addition, data provided by the same study indicated that TPO-Ab (+) women who did not achieve pregnancy were characterized by higher TSH levels prior to IVF treatment. In a study in 2014 published by Mintzori, et al. [16], 15 euthyroid TAA (+) women were compared to 67 euthyroid TAA (-) women in respect to the IVF outcome. No statistically significant difference could be established regarding clinical pregnancy rate (33.3\% vs. $37.3 \%)$, live birth rate $(26.7 \%$ vs. $34.3 \%)$ and miscarriage rate $(6.7 \%$ vs. $3 \%)$. In a retrospective study published in 2013, Magri, et al. [15] highlighted that the presence of the TAA did not affect clinical pregnancy rate. Nonetheless, the performance regarding ovarian stimulation was significantly poorer when TAA (+) women were compared to TAA (-) women. In addition, data provided by this study indicated that the presence of TAA could negatively affect the short-term outcomes of the IVF treatment, only in women with TSH levels $>2.5 \mathrm{mlU} / \mathrm{L}$. Authors underlined that the presence of TAA coupled by TSH levels $>2.5 \mathrm{mIU} / \mathrm{L}$ could impair IVF outcome; thus they suggest that all women should be screened for TAA and TSH levels prior to IVF treatment. In the retrospective study of Chai, et al. [10], 511 TAA (-) women were compared to 125 TAA (+) women. In both groups, a number of patients were diagnosed with subclinical hypothyroidism (TSH > $2.5 \mathrm{mIU} / \mathrm{L}$ ). Thus, the study population was divided in four groups namely, TAA (-), euthyroid and/or subclinical hypothyroidism, and TAA (+), euthyroid and/or subclinical hypothyroidism. No statistically significant difference could be established pertaining to the patients' age, the menstrual cycle hormonal profile, the etiology of infertility, the clinical pregnancy rate, the live birth rate, and the miscarriage rate among the four groups. Data presented in this study suggest that live birth rate and clinical pregnancy rate are not impaired in women with TAA, and/ or subclinical hypothyroidism. However, the observation that the subclinical hypothyroidism is not correlated with an impaired IVF outcome is not confirmed by various other studies [7]. In contrast to the abovementioned studies, Zhong, et al. [22] and Revelli, et al. [19] highlighted that the presence of the TAA could affect negatively the IVF outcome. In the study of Zhong, et al. [22], 90 TAA (+) positive women were compared to 676 TAA (-) women with respect to the IVF outcome. 
No statistically significant difference could be established regarding the patients' age, the hormonal profile (FSH, LH, and E2 levels), the response to ovarian stimulation and the number of oocytes retrieved among the two groups. TAA (+) women presented with statistically significant lower fertilization rate, implantation rate, and clinical pregnancy rate and higher miscarriage rate when compared with TAA (-) women. With regards to these data, the authors suggested that the presence of TAA in women undergoing IVF could negatively affect the IVF outcome. However, the lack of information regarding the hormonal profile of thyroid functionality (the TSH levels and the free T4 levels were not presented) poses a major limitation characterizing this study and adding to a high risk of bias. Therefore, no evidence can extract with safety. In the study published by Revelli, et al. [19], 129 TAA (+) euthyroid women were divided into three groups according to the adjuvant treatment patients received during the IVF cycle. The first group consisted of TAA (+) euthyroid women without adjuvant treatment (38 women), the second group consisted of TAA (+) euthyroid women with adjuvant treatment with LT4 (55 women), while the third group consisted of TAA $(+)$ euthyroid women with adjuvant treatment with LT4, acetyl-salicylic acid and prednisolone (36 women). Two hundred TAA (-) euthyroid women were included in the study as a control group. The TAA (+) euthyroid women without adjuvant treatment were presented with a significantly lower number of oocytes retrieved following control ovarian stimulation, significantly lower implantation rate and significantly higher abortion rate compared with controls. In contrast, the TAA (+) euthyroid women who were treated with adjuvant treatment employing LT4, acetyl-salicylic acid and prednisolone, exhibited a significantly better overall performance in the IVF cycle similar to the performance observed in the control group. Authors highlighted that the LT4, acetyl-salicylic acid and prednisolone administration could be beneficial for euthyroid TAA (+) women undergoing IVF treatment. Due to the retrospective design and the small number of participants included this study would not be assessed as clear of bias. Consequently, the study's outcomes should be verified by large prospective cohort studies with an equal number of participants in each group.

\section{Discussions}

It is well documented in the literature that thyroid pathogenesis could negatively affect-via several mechanisms- functionality of the female reproductive system. Overt thyroid diseases, such as Hashimoto thyroiditis, are strongly correlated with menstrual irregularities, anovulation, infertility, and poor obstetric outcomes. The main pathogenic mechanism leading to thyroid dysfunction among the women of reproductive age is thyroid autoimmunity. The infertile women present with a higher prevalence of thyroid autoimmune dysfunction in comparison to the general population. However, there are insufficient data regarding the direct impact of TAA positivity on IVF outcome, especially regarding the specific population consisting of infertile, euthyroid TAA positive women. Following evaluation of the data published in literature from 2006 to 2018, TAA positivity in euthyroid women does not appear to be associated with adverse outcomes following IVF/ICSI cycles, as demonstrated by the great majority of the studies analyzed herein. Regarding the miscarriage rates, no safe conclusions can yet be reached. Even though the majority of the studies do not report any statistically significant difference, a meta-analysis observed higher miscarriage rates in TAA positive women [23]. Data presented in this review seem to follow a trend of higher miscarriage rates in euthyroid TAA positive women in comparison to TAA negative women. This trend has also been noted in a recent meta-analysis published by Busnelli, et al. [24]. Nonetheless, the maternal TAA positivity could impair IVF/ICSI outcomes when overt thyroid dysfunction or subclinical hypothyroidism are coexistent. It is possible that TAA does not affect directly oocyte development, embryo quality, and embryo implantation, but rather compromise the implantation physiological processes through Th1 mediated cytotoxic phenomena [25]. A limitation in safely extracting conclusions is the high variability between the studies regarding infertility etiology and inclusion/exclusion criteria. Thus, the level of heterogeneity among them is evaluated as being notably high. Another reason for caution is the different protocols employed both for IVF/ICSI treatment. The small size of the studies, especially when compared to the control group, limit the strength of the studies and of the data provided. Larger and better-designed studies are a requirement in order to provide higher quality evidence. The present study's conclusions are concordant to other studies' in the field $[25,26]$ and in agreement with the current Guidelines of the American Thyroid Association [7]. Results sourced by the current critical analysis attempted in this review article lead to the conclusion that women positive for thyroid autoantibodies should not be considered as subject to an additional risk regarding IVF failure through the respective associations. Such a conclusion could extend to affect decision making and management of euthyroid women with respect to rendering screening for thyroid autoantibodies prior to IVF redundant. Further to that, and as recommended by current guidelines [7], euthyroid women positive for thyroid autoantibodies should not be treated with adjustment glucocorticoid treatment prior to the IVF cycle in order to increase success rates.

\section{References}

1. Feuer S, Rinaudo P (2016) From Embryos to Adults: A $\mathrm{DOHaD}$ Perspective on In Vitro fertilization and other assisted reproductive technologies. Healthcare (Basel) 4.

2. Doufas AG, Mastorakos G (2000) The hypothalamicpituitary-thyroid axis and the female reproductive system. Ann N Y Acad Sci 900: 65-76. 
3. Quintino-Moro A, Zantut-Wittmann DE, Tambascia M, Machado H da C, Fernandes A (2014) High Prevalence of Infertility among Women with Graves' Disease and Hashimoto's Thyroiditis. Int J Endocrinol 2014: 6.

4. Unuane D, Velkeniers B, Deridder S, Bravenboer B, Tournaye $\mathrm{H}$, et al. (2016) Impact of thyroid autoimmunity on cumulative delivery rates in in vitro fertilization/ intracytoplasmic sperm injection patients. Fertil Steril 106: 144-150.

5. Unuane D, Velkeniers B, Anckaert E, Schiettecatte J, Tournaye $\mathrm{H}$, et al. (2013) Thyroglobulin autoantibodies: is there any added value in the detection of thyroid autoimmunity in women consulting for fertility treatment? Thyroid 23: 1022-1028.

6. Poppe K, Velkeniers B, Glinoer D (2007) Thyroid disease and female reproduction. Clin Endocrinol (Oxf) 66: 309-321.

7. Alexander EK, Pearce EN, Brent GA, Brown RS, Chen $\mathrm{H}$, et al. (2017) 2017 Guidelines of the American Thyroid Association for the Diagnosis and Management of Thyroid Disease During Pregnancy and the Postpartum. Thyroid 27: 315-389.

8. Ferraretti AP, Nygren K, Andersen AN, de Mouzon J, Kupka $M$, et al. (2017) Trends over 15 years in ART in Europe: An analysis of 6 million cycles. Hum Reprod Open 2.

9. Caccavo D, Pellegrino NM, Nardelli C, Vergine S, Leone L, et al. (2016) Anti-laminin-1 antibodies in serum and follicular fluid of women with Hashimoto's thyroiditis undergoing in vitro fertilization. Int J Immunopathol Pharmacol 29: 280287.

10. Chai J, Yeung W-YT, Lee C-YV, Li H-WR, Ho P-C, et al. (2014) Live birth rates following in vitro fertilization in women with thyroid autoimmunity and/or subclinical hypothyroidism. Clin Endocrinol (Oxf) 80: 122-127.

11. Chen X, Mo M-L, Huang C-Y, Diao L-H, Li G-G, et al. (2017) Association of serum autoantibodies with pregnancy outcome of patients undergoing first IVF/ICSI treatment: A prospective cohort study. J Reprod Immunol 122: 14-20.

12. Karacan M, Alwaeely F, Cebi Z, Berberoglugil M, Batukan $M$, et al. (2013) Effect of antithyroid antibodies on ICSI outcome in antiphospholipid antibody-negative euthyroid women. Reprod Biomed Online 27: 376-380.

13. Kilic S, Tasdemir N, Yilmaz N, Yuksel B, Gul A, et al. (2008) The effect of anti-thyroid antibodies on endometrial volume, embryo grade and IVF outcome. Gynecol Endocrinol 24: 649-655.

14. Lukaszuk K, Kunicki M, Kulwikowska P, Liss J, Pastuszek E, et al. (2015) The impact of the presence of antithyroid antibodies on pregnancy outcome following intracytoplasmatic sperm injection-ICSI and embryo transfer in women with normal thyreotropine levels. J Endocrinol Invest 38: 1335-1343.
15. Magri F, Capelli V, Gaiti M, Brambilla E, Montesion L, et al. (2013) Impaired outcome of controlled ovarian hyperstimulation in women with thyroid autoimmune disease. Thyroid 23: 1312-1328.

16. Mintziori G, Goulis DG, Gialamas E, Dosopoulos K, Zouzoulas D, et al. (2014) Association of TSH concentrations and thyroid autoimmunity with IVF outcome in women with TSH concentrations within normal adult range. Gynecol Obstet Invest 77: 84-88.

17. Monteleone $\mathrm{P}$, Parrini D, Faviana $\mathrm{P}$, Carletti E, Casarosa E, et al. (2011) Female infertility related to thyroid autoimmunity: the ovarian follicle hypothesis. Am J Reprod Immunol 66: 108-114.

18. Negro R, Formoso G, Coppola L, Presicce G, Mangieri T, et al. (2007) Euthyroid women with autoimmune disease undergoing assisted reproduction technologies: the role of autoimmunity and thyroid function. J Endocrinol Invest 30: 3-8.

19. Revelli A, Casano S, Piane LD, Grassi G, Gennarelli G, et al. (2009) A retrospective study on IVF outcome in euthyroid patients with anti-thyroid antibodies: Effects of levothyroxine, acetyl-salicylic acid and prednisolone adjuvant treatments. Reprod Biol Endocrinol 7: 137.

20. Sakar MN, Unal A, Atay AE, Zebitay AG, Verit FF, et al. (2016) Is there an effect of thyroid autoimmunity on the outcomes of assisted reproduction? J Obstet Gynaecol 36: 213-217.

21. Tan S, Dieterle S, Pechlavanis S, Janssen OE, Fuhrer D (2014) Thyroid autoantibodies per se do not impair intracytoplasmic sperm injection outcome in euthyroid healthy women. Eur J Endocrinol 170: 495-500.

22. Zhong Y, Ying Y, Wu H, Zhou C, Xu Y, et al. (2012) Relationship between antithyroid antibody and pregnancy outcome following in vitro fertilization and embryo transfer. Int J Med Sci 9: 121-125.

23. Toulis KA, Goulis DG, Venetis CA, Kolibianakis EM, Negro R, et al. (2010) Risk of spontaneous miscarriage in euthyroid women with thyroid autoimmunity undergoing IVF: A meta-analysis. Eur J Endocrinol 162: 643-652.

24. Busnelli A, Paffoni A, Fedele L, Somigliana E (2016) The impact of thyroid autoimmunity on IVF/ICSI outcome: A systematic review and meta-analysis. Hum Reprod Update 22: $775-790$.

25. He H, Jing S, Gong F, Tan YQ, Lu GX, et al. (2016) Effect of thyroid autoimmunity per se on assisted reproduction treatment outcomes: A meta-analysis. Taiwan J Obstet Gynecol 55: 159-165.

26. Deroux A, Dumestre-Perard C, Dunand-Faure C, Bouillet L, Hoffmann P (2017) Female Infertility and Serum Autoantibodies: A Systematic Review. Clin Rev Allergy Immunol 53: 78-86. 